\title{
Modulated Light Curves of Multiperiodic Stars
}

\section{József M. Benkő, Róbert Szabó and Margit Paparó}

Konkoly Observatory of the Hungarian Academy of Sciences, H-1121 Budapest, Hungary email: benko@konkoly.hu

\begin{abstract}
We modelled the light curves of modulated multiperiodically pulsating stars by a simple double-mode pulsation with combined amplitude and frequency modulations. The synthetic light curves and their spectra show similar features to those we found and discussed for monoperiodic stars. Comparing the synthetic light curves and their spectra with the observed ones helps us to classify the modulations and to distinguish between long-period modulation (Blazhko effect) and the other types of amplitude and/or period changes.
\end{abstract}

Keywords. methods: analytical, stars: oscillations, stars: variables: other

\section{Introduction}

Benkő et al. (2011) described the light variations of Blazhko RR Lyræ stars by a formalism adopted from the theory of electronic signals. In our treatment we assumed monoperiodic light curves to be carrier waves and that they became modulated in either their amplitude (AM), or their frequency (FM), or both. That approach shows numerous advantages compared to the traditional Fourier sums. Since it is general enough, it can be extended to many types of modulated light curves. Here we present some results based on a synthetic light curve generated by a double-periodic carrier signal.

Our present study was motivated by recent publications in which the Blazhko effect was discussed in connection with different multiperiodically pulsating stars. Papers by Henry et al. (2005), Moskalik \& Kołaczkowski (2009) and Breger (2010) raised the possibility of modulations (Blazhko effect) in $\gamma$ Dor, beat Cepheids and $\delta$ Scuti stars, respectively. A further development is the recent discovery that RR Lyræ stars can pulsate in more than one mode (occasionally or permanently) and can show simultaneous Blazhko effects (Poretti et al. 2010, Benkő et al. 2010).

\section{The Model}

To demonstrate how our method works for multiperiodic light curves we chose the simplest multiperiodic (visual double-mode) pulsation as a test case. Let the carrier wave be modelled by a light curve, $c(t)$ :

$$
c(t)=c_{0}(t) c_{1}(t), \quad \text { where } \quad c_{k}(t)=a_{0 k}+\sum_{i_{k}}^{n_{k}} a_{i k} \sin \left(2 \pi i_{k} f_{k} t+\varphi_{i k}\right) .
$$

$n_{k}=1,2,3, \ldots ; k=0,1$ integers. In practice, we used a synthetic RRd (= doublemode RR Lyræ) light curve. A signal that is simultaneously modulated in amplitude and frequency can be written formally as

$$
m_{\mathrm{comb}}(t)=\left[1+m_{\mathrm{mod}}^{\mathrm{A}}(t)\right] \tilde{c}(t) .
$$




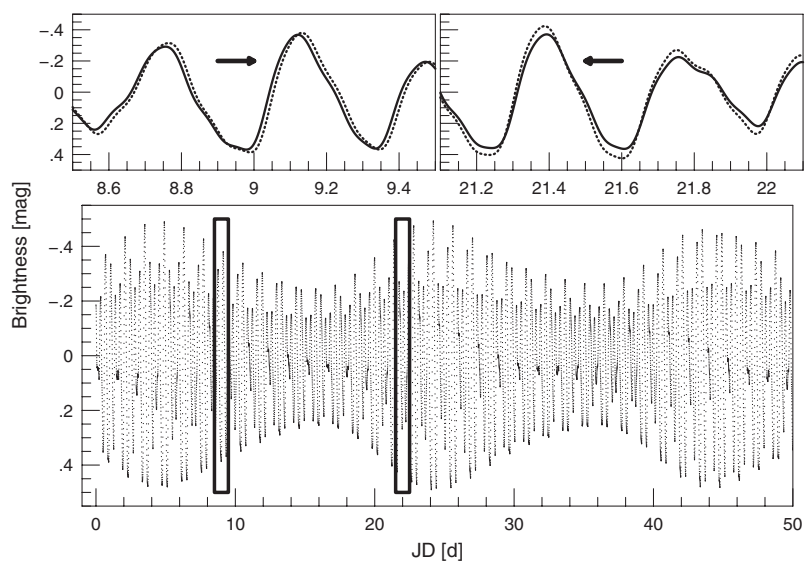

Figure 1. A double-periodic light curve modulated in both amplitude and frequency (bottom). Different non-sinusoidal periodic functions were used for modulations. Manifestation of the phase variation is shown in the top panels, where the modulated light curve (dotted) is compared to the unmodulated carrier one (continuous). The phase shifts are indicated by arrows.

Here $\tilde{c}(t)$ is the same expression as Eq. (2.1), but we substitute functions $\tilde{f}_{k}(t)=f_{k}+$ $m_{\bmod }^{\mathrm{F}}(t)$ instead of the fixed frequencies $f_{k}$ for all $k=0,1$. The modulation functions $m_{\text {mod }}^{\mathrm{A}}(t), m_{\text {mod }}^{\mathrm{F}}(t)$ are assumed to be periodic and can be represented by finite Fourier sums. Fig. 1 compares the artificial light curve to the carrier light curve of Eq. (2.1).

\section{Characteristics of the Light Curve and the Fourier Spectrum}

Some features are evident directly from the light curve in Fig. 1. Since the AM is assumed to be non-sinusoidal the envelope curve shows a horizontally asymmetric shape (bottom panel), while the FM indicates phase variations (top panels).

Additional investigations can be carried out in the Fourier spectrum (Fig. 2). The spectrum is dominated by the two main frequencies, $f_{0}$, and $f_{1}$ and their different number of harmonics $\left(i_{k} f_{k}, i_{k}=1,2, \ldots, n_{k} ; k=0,1\right)$, and their linear combinations constitute the carrier wave spectrum. The appearance of the linear combination frequencies is a wellknown observed feature of double-mode RR Lyræ stars. Assumption (2.1) provides those frequencies automatically. When we produced the synthetic light curve we represented the non-sinusoidal AM function $m_{\mathrm{mod}}^{\mathrm{A}}(t)$ as a Fourier sum with two components, so the frequency $f_{\mathrm{m}}$ and its harmonic $2 f_{\mathrm{m}}$ consequently appear in the resultant spectrum (insert in Fig. 2). FM modulation - even in the sinusoidal case - produces an infinite number of side peaks (see Szeidl \& Jurcsik 2009, Benkö et al. 2011) around the main frequencies and their harmonics. Those multiplets $\left(i_{k} f_{k} \pm l f_{\mathrm{m}}\right)$ can be identified in the top panels in Fig. 2 . It is important to note that more than two side peaks are seen $(l>2)$ in each multiplet. That shows direct evidence of FM; otherwise, the case can not be separated from a pure non-sinusoidal AM. An additional feature also suggests FM: the higher-order harmonics have more detectable side frequencies than the lower-order ones (compare the top left and right panels of Fig. 2). That distinguishes FM from the very similar phase modulation (PM). Combined AM+FM modulation results in highly non-symmetrical amplitudes of the side peaks. Some side peaks are almost missing, as is shown by the vertical lines at their positions (top right panel, Fig. 2). Those and some additional features have been discussed in detail by Benkő et al. 2011), where single period carrier waves were used. The significant difference between that and the present work is the appearance 


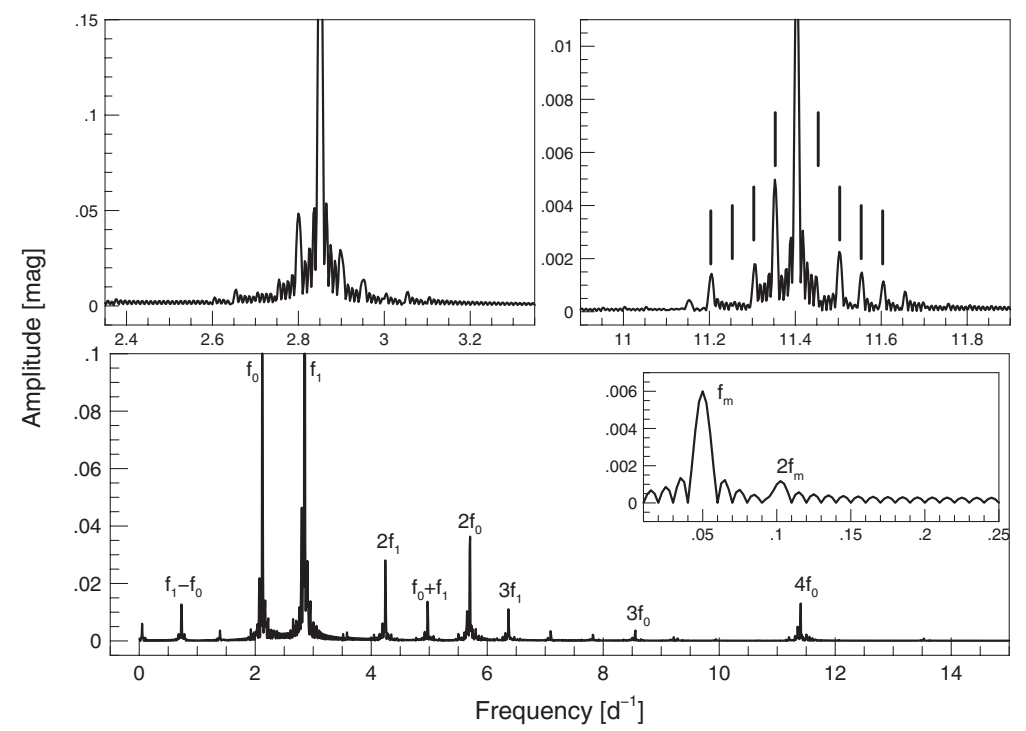

Figure 2. Fourier amplitude spectrum of the synthetic modulated light curve in Fig. 1 (bottom main panel) and its zoomed segments around $f_{0}$ (top left panel), $4 f_{0}$ (top right panel-vertical lines show the positions of the side peaks) and $f_{\mathrm{m}}$ (insert in bottom panel).

of linear combination frequencies. By investigating the surroundings of the combination frequencies we find multiplet patterns around all linear combination frequencies that are similar to those around the main frequencies and their harmonics.

All of the phenomena which we have found by this treatment can be explained by the mathematics of the modulation. Conversely, if an observed light curve does not show those key features (see also Benkö et al. 2011) we have to find a different origin for the amplitude and/or phase variations.

\section{Acknowledgements}

The authors acknowledge the support of ESA PECS projects Nos. 98114 \& 4000103541.

\section{References}

Benkő, J. M., Kolenberg, K., Szabó, R., Kurtz, D. W., \& Bryson, S. et al. 2010, MNRAS, 409, 1244

Benkő, J. M., Szabó, R., \& Paparó, M. 2011, MNRAS, 417, 974

Breger, M. 2010, in: Sterken C., Samus N., \& Szabados L. (eds.), Variable Stars, the Galactic Halo and Galaxy Formation (Moscow: Sternberg Astron. Inst.), p. 95

Chadid, M., Benkő, J. M., Szabó, R., Paparó, M., \& Chapellier, E. et al. 2010, A\&̛A, 510, A39

Guggenberger, E., Kolenberg, K., Chapellier, E., Poretti, E., \& Szabó, R. et al. 2011, MNRAS, 415,1577

Henry, G. W., Fekel, F. C., \& Henry, S. M. 2005, AJ, 129, 2815

Moskalik, P. \& Kołaczkowski, Z. 2009, MNRAS, 394, 1649

Poretti, E., Paparó, M., Deleuil, M., Chadid, M., \& Kolenberg, K. et al. 2010, A\& A, 520, A108

Szeidl, B. \& Jurcsik, J. 2009, CoAst, 160, 17 